\title{
Traumatic Intracranial Hematomas: Prognostic Value of Contrast Extravasation
}

\author{
L. Letourneau-Guillon, T. Huynh, R. Jakobovic, R. Milwid, S.P. Symons, and R.I. Aviv
}

\section{ABSTRACT}

BACKGROUND AND PURPOSE: Contrast extravasation within spontaneous intracranial hemorrhage is a well-described predictor of hematoma growth, poor clinical outcome, and mortality. The purpose of this study was to assess the prognostic value of contrast extravasation in acute traumatic intracranial hematomas.

MATERIALS AND METHODS: In our institution, CTA (including PCCT) is the primary screening technique for cervical vascular injuries. Sixty consecutive patients with at least 1 acute intracranial hematoma ( $\mathrm{ICH}$, subdural hematoma, and/or epidural hematoma) meeting predefined size criteria, with CTA/PCCT performed within 24 hours of admission and follow-up CT within 72 hours of admission, were retrospectively evaluated for CE by 2 observers. The predictive value of CE for a composite outcome (hematoma expansion, need for hematoma evacuation, in-hospital mortality) was evaluated on a per-patient basis. Interobserver agreement for CE and the association between baseline variables and outcome were also examined. Different patterns of extravasation were evaluated on a per-lesion basis, with outcomes including hematoma expansion and evacuation.

RESULTS: CE was present in $30(50 \%)$ patients with almost perfect interobserver agreement $(\kappa=0.87 ; 95 \% \mathrm{Cl}, 0.74-0.99)$. The per-patient multivariate analysis showed independent association of midline shift $(P=.020)$, Glasgow Coma Scale score $\leq 8(P=.024)$, and CE $(P=.017)$, with poor outcome and demonstrated a trend toward poor outcome prediction for age 65 years or older $(P=.050)$. In the per-lesion analysis, only extravasation identified on CTA (active and contained extravasation) was associated with hematoma expansion and evacuation.

CONCLUSIONS: Contrast extravasation within intracranial hematomas predicts poor in-hospital outcome in the setting of acute traumatic intracranial injuries.

ABBREVIATIONS: $\mathrm{CE}=$ contrast extravasation; $\mathrm{Cl}=$ confidence interval; $\mathrm{ICH}=$ intracerebral hematoma; $\mathrm{OR}=$ odds ratio; $\mathrm{PCCT}=$ postcontrast $\mathrm{CT} ; \mathrm{PCL}=$ postcontrast leakage

$\mathbf{T}$ raumatic brain injury is a major health and socioeconomic problem worldwide and is a leading cause of mortality and disability. ${ }^{1}$ NCCT plays a central role in the triage of patients with acute head injuries, given its high sensitivity for the detection of hemorrhagic lesions. In the presence of acute intracranial hematomas, management options include emergent surgical evacua-

Received March 5, 2012; accepted after revision July 21.

From the Division of Neuroradiology, Department of Medical Imaging, Sunnybrook Health Sciences Centre, University of Toronto, Toronto, Ontario, Canada.

Paper previously presented in part at: 50th Annual Meeting of the ASNR and the Foundation of the ASNR Symposium, April 21-26, 2012; New York, New York.

Please address correspondence to Richard Aviv, MD, Sunnybrook Health Science Centre, Room AG 31, 2075 Bayview Ave, Toronto, ON, M4N 3M5, Canada; e-mail: richard.aviv@sunnybrook.ca

三 Indicates article with supplemental on-line tables.

Indicates article with supplemental on-line figure.

http://dx.doi.org/10.3174/ajnr.A3309 tion or close observation including imaging surveillance. Sixteen percent to $65 \%$ of the patients in the latter group will show hematoma progression, ${ }^{2-8}$ and $13 \%-19 \%$ will require delayed surgery $^{2-4}$; hematoma expansion is also associated with increased morbidity and mortality. ${ }^{6,7}$ Despite previous identification of multiple NCCT predictors of hematoma expansion, ${ }^{2,3,5,6,9}$ this modality remains inherently limited for the detection of active hemorrhage. ${ }^{10}$

CTA is widely used as the initial screening technique for the detection of traumatic vascular injury. ${ }^{11}$ In nontraumatic ICHs, contrast extravasation on CTA has been shown to predict hematoma expansion, poor outcome, and mortality. ${ }^{12-23}$ Despite CT reports dating back $>30$ years identifying contrast extravasation in traumatic head injuries, ${ }^{24}$ there has been limited analysis of this sign in the setting of trauma. A recent study evaluated 22 patients with traumatic cerebral contusions and concluded that early parenchymal $\mathrm{CE}$ is associated with clinical progression, cerebral 
Table 1: Patterns of extravasation

\begin{tabular}{lll}
\hline \multirow{2}{*}{ Descriptive Term } & \multicolumn{2}{c}{$\begin{array}{c}\text { Extravasation Presence } \\
\text { and Pattern }\end{array}$} \\
\cline { 2 - 3 } Contained extravasation & CTA & \multicolumn{1}{c}{ PCCT } \\
Active extravasation & $\begin{array}{l}\text { Presence } \\
\text { PCL }\end{array}$ & Not expanding \\
CE & Absence & Expanding \\
\hline
\end{tabular}

edema, clinical deterioration, and the need for subsequent surgery. $^{25}$

The aim of this study was to analyze the value of contrast extravasation for the prediction of hematoma progression or evacuation as well as the prediction of in-hospital mortality. In addition, spontaneous ICH studies have shown the potential importance of assessing the rate of contrast extravasation, which can be grossly estimated by using a biphasic acquisition protocol. ${ }^{15,26}$ Therefore, we also examined the prognostic value of different patterns of extravasation (contained extravasation, active extravasation, and postcontrast leakage; Table 1), according to their appearance on a CTA/PCCT protocol.

\section{MATERIALS AND METHODS Patient Selection}

This retrospective study was ethics board-approved. Patients presenting with head and/or neck injury to our level 1 regional trauma center frequently undergo cranial NCCT. This is supplemented by head and neck CTA to screen for cervical vascular injury at the discretion of the treating physician and is based on the modified Denver Criteria, ${ }^{11}$ which include clinical or neuroimaging evidence of ischemic infarction, cervical hematoma/softtissue injury, and craniocervical fractures, among others. All consecutive patients with head trauma with at least 1 acute traumatic ICH (including contusion, shear injury, or penetrating injury) of $\geq 10 \mathrm{~mm}$ in the largest transverse diameter or acute extra-axial hematoma (subdural hematoma or epidural hematoma) of $\geq 2$ $\mathrm{mm}$ in thickness and with CTA performed within 24 hours of admission and with follow-up head CT within 72 hours of admission were included.

\section{Patient Cohort}

Between July 2007 and August 2011, two hundred four patients presenting with traumatic head injury were evaluated by CTA to screen for cervical vascular injury. Of these, 144 were excluded (lack of intracranial hematoma in 91, hematoma smaller than the predefined cutoff values in 22, CTA performed $>24$ hours after admission in 13, lack of CT follow-up within 72 hours of admission in 16 due to transfer to an outside hospital, nondiagnostic CTA in 2 patients). Patients in whom CT follow-up was unavailable because of emergent surgical hematoma drainage or in-hospital death were included because these 2 end points were part of the per-patient composite outcome (see "Statistical Analysis").

\section{Clinical Data}

Patient charts were reviewed for age, sex, mechanism of injury, time of injury, presence of significant extracranial injuries, admission Glasgow Coma Scale score, admission blood pressure, time of CTA/PCCT and follow-up CT, need for hematoma evacuation, and in-hospital mortality. Medical history, including a history of hypertension, diabetes mellitus, bleeding diathesis, and antiplatelet or anticoagulation use, was recorded. Admission laboratory values recorded included platelet count, international normalized ratio, partial thromboplastin time, and serum glucose levels.

\section{Image Acquisition}

All studies were performed on a 64-section CT scanner (LightSpeed VCT; GE Healthcare, Milwaukee, Wisconsin). NCCTs were generally performed immediately following admission once the trauma team stabilized the patient. CTA of the head and neck and PCCT of the head were performed either immediately following the NCCT or later following admission, depending on the clinical setting. PCCT of the head routinely follows every head and neck CTA at our institution. Pre- and postcontrast head imaging were performed by using the same technical parameters and covering from the skull base to the vertex as follows: $120 \mathrm{kVp} ; 340$ $\mathrm{mA} ; 4 \times 5 \mathrm{~mm}$ collimation; 1 s/rotation; 5-mm section thickness; table speed, $15 \mathrm{~mm} /$ rotation. Helical CTA studies were obtained from $\mathrm{C} 7$ to the vertex. CTA covered from the aortic arch to the vertex; parameters were $0.7-\mathrm{mL} / \mathrm{kg}$ iohexol $(300-\mathrm{mg} \mathrm{I} / \mathrm{mL}$ concentration), maximum of $90 \mathrm{~mL}$ through an antecubital vein via an 18 - or 20 -ga angiocatheter followed by a $40-\mathrm{mL}$ saline bolus; $5-\mathrm{mL} / \mathrm{s}$ injection rate; injection triggered by using a bolus-tracking method; $120 \mathrm{kV}$ (peak); $270 \mathrm{~mA} ; 1$ s/rotation; 0.0625 - to $1.25-\mathrm{mm}$ section thickness; table speed, $3.75 \mathrm{~mm} /$ rotation. CT technologists performed all postprocessing, including multiplanar reformations, at the CT operator's console. Coronal and sagittal multiplanar reformat images were created as 7-mm-thick images spaced by $3 \mathrm{~mm}$. Studies were viewed on PACS workstations.

\section{Imaging Analysis/Interpretation}

Initial NCCTs were evaluated for intracranial hematoma type (traumatic intracerebral hematoma, subdural hematoma, epidural hematoma). Up to 9 hematomas were evaluated per patient (allowing up to 4 traumatic intracerebral hematomas, 3 subdural hematomas, and 2 epidural hematomas per patient; the largest lesions were considered if there were $>9$ hematomas in a given patient). NCCTs were also assessed for subarachnoid hemorrhage presence, maximal SAH thickness, and IVH presence graded according to the score of Graeb et al. ${ }^{27}$ This score is a 12-point grading system based on the amount and distension of the 4 ventricles by IVH. Septum pellucidum shift was measured at the level of the foramen of Monro. ${ }^{28}$

CTA and PCCT studies were independently evaluated for extravasation by 1 neuroradiologist (R.I.A.) and a neuroradiology fellow (L.L.-G.), both blinded to the outcomes. All contrast-enhanced studies were evaluated in conjunction with the concurrent noncontrast CT to avoid extravasation mimics, ${ }^{29}$ including the presence of osseous fragments secondary to cranial fractures. We used a modified version of the "spot sign" criteria (On-line Table $1)^{30}$ to diagnose extravasation but allowed the possibility of a connection to a vessel outside the hematoma only for extra-axial hematomas. If such an apparent connection was found, the presumed extravasation focus was considered positive if the size and morphology were different from those in the parent vessel and if it 
appeared as a blind-ended focus. Furthermore, if present, expansion of the focus of extravasation between the CTA and PCCT was also supportive of the diagnosis of extravasation. For the purpose of the per-lesion analysis, we defined 3 patterns of enhancement as listed in Table 1. These include contained extravasation, active extravasation, and postcontrast leakage. For the purpose of this study, "contrast extravasation" refers to any type of these 3 patterns. The number of extravasation foci per hematoma was also noted. For the subsequent analyses, consensus was used for the presence and number of foci of extravasation.

Computer-aided planimetry was used to measure the volume of all hematomas on both the initial CTA study and the next available follow-up CT by using the Medical Image Processing, Analysis, and Visualization software (Center for Information Technology, National Institutes of Health, Bethesda, Maryland). An increase of hematoma size of $>12 \mathrm{~mL}$ and $>33 \%$ was considered significant both for traumatic intracerebral hematomas and extra-axial hematomas.

\section{Statistical Analysis}

The interobserver agreement for number and presence of extravasation was calculated with the $\kappa$ statistic. Values of $\kappa$ of $0.21-0.4$, $0.41-0.6,0.61-0.8$, and $0.81-1$ were considered fair, moderate, substantial, and near-perfect, respectively.

For the per-patient analysis, we used a composite outcome including at least 1 of the following: hematoma expansion, hematoma evacuation, or in-hospital mortality. We only evaluated the presence of any type of extravasation without attempting to characterize the different patterns because $>1$ pattern could be found in any given patient (who often harbored $>1$ hematoma). We summed all hematoma volumes for each individual patient. Association between baseline variables and both the presence of CE and the outcomes were examined. Thresholds for age $>65$ years, ${ }^{31}$ glucose level $>8.3 \mathrm{~mm} / \mathrm{L},{ }^{14,32}$ Glasgow Coma Scale score $\leq 8,9,33$ and midline shift $\geq 5 \mathrm{~mm}^{34-36}$ were selected on the basis of previous data and clinical relevance. Continuous data were assessed by using the Student $t$ test or Wilcoxon rank-sum test, on the basis of normality of data by using the Shapiro-Wilk test. Categoric data were assessed by using the Fisher exact test. Multivariable logistic regression was performed for associations with the combined outcome. Variable selection was based on clinical and statistical significance in addition to model fit by using the Akaike Information Criterion and nested models. Final multivariable model calibration and discrimination were examined by using the Hosmer and Lemeshow $\chi^{2}$ statistic and area under the receiver operating characteristic curve. To improve regressionmodel prediction, we adjusted parameter estimates by using uniform shrinkage. ${ }^{37}$

For the per-lesion analysis, we examined 3 different outcomes: hematoma expansion, need for hematoma evacuation, and the combination of these 2 end points. Patient clustering was controlled for by use of generalized estimating equations. Diagnostic performance values with exact confidence intervals were adjusted for patient clustering.

$P<.05$ was considered statistically significant. All analyses were performed by using SAS 9.2 (SAS Institute, Cary, North
Carolina) and R, Version 2.13.2 (http://cran.r-project.org/bin/ windows/base/old/2.13.2/).

\section{RESULTS}

Sixty patients, including 46 (77\%) men, harboring a total of 138 intracranial hematomas, were enrolled. Head trauma causes included fall in 34 (57\%), motor vehicle collision in 22 (37\%), and direct impact in $4(7 \%)$ patients, including 1 instance of penetrating head injury.

\section{Contrast Extravasation}

An example of contrast extravasation is shown in On-line Figure 1. CE was present in $30 / 60(50 \%)$ patients with a median (interquartile range) number of 3 foci (1-5) per patient. The interobserver agreement for $\mathrm{CE}$ presence and the number of foci was nearly perfect $(\kappa=0.87 ; 95 \%$ CI, $0.74-0.99$ and $\kappa=0.85 ; 95 \%$ CI, $0.80-0.91$, respectively). Associations between baseline characteristics and the presence of CE are shown in On-line Table 2. In this univariate analysis, CE was associated with hematoma expansion, greater initial total hematoma volume, midline shift $\geq 5$ $\mathrm{mm}$, death, and shorter trauma-to-CTA time compared with patients without CE. There was a nonsignificant trend toward increased subdural hematoma volume in patients with CE (median [interquartile range]: volume $21.1 \mathrm{~mL}$ [5.1-64.1] versus $6.2 \mathrm{~mL}$ [3.3-9.3]; $P=.081$ ). The Graeb score for intraventricular hemorrhage also showed a trend toward a higher score in the group showing $\mathrm{CE}(P=.09)$. No patient had a history of bleeding diathesis. Antiplatelet $(P=1.00)$ or anticoagulation $(P=.49)$ medication use was similar in the 2 groups.

\section{Patient Outcomes}

Twenty-six (43\%) patients had a poor outcome, including 7 instances of hematoma expansion, 9 hematoma evacuations, and 17 in-hospital mortalities. Of these, 5 patients had surgical evacuation and 7 patients died before imaging follow-up. Hematoma expansion occurred before in-hospital death in 4 patients, hematoma evacuation occurred before death in 1 patient, and 1 patient had all 3 outcomes (hematoma expansion, evacuation, and inhospital death). On-line Table 3 demonstrates univariate associations between baseline variables and the primary composite end point. Age $>65$ years, glucose level $>8.3 \mathrm{mmol} / \mathrm{L}$, total admission hematoma volume, admission Glasgow Coma Scale score $\leq 8$, admission midline shift $\geq 5 \mathrm{~mm}$, and shorter trauma-to-CTA time were all associated with poor outcome. The presence of CE was also significantly associated with poor outcome (OR, 8.00; 95\% CI, 2.47-25.9; $P<.001)$. There was a trend toward lower platelet count and IVH presence in patients with poor outcome. Antiplatelet or anticoagulation use, blood pressure, extracranial injuries, hematoma type, SAH presence, IVH presence, and Graeb score for IVH were not associated with poor outcome. Extravasation remained significant when controlling for the other variables individually with the exception of initial total hematoma volume for which extravasation showed a trend toward significance (OR, 3.54; 95\% CI, 0.965-12.960; $P=.057)$. Because hematoma volume and midline shift were collinear and midline shift was a stronger predictor of the outcome, only the latter was included in the multivariate analysis. In the multivariate analysis, contrast 
Table 2: Multivariable logistic regression model for prediction of combined outcome

\begin{tabular}{lcccc}
\hline \multicolumn{1}{c}{ Variable } & OR & $\begin{array}{c}\text { Lower } \\
\mathbf{9 5 \% ~ C l}\end{array}$ & $\begin{array}{c}\text { Upper } \\
\mathbf{9 5 \% ~ C l}\end{array}$ & $\begin{array}{c}\boldsymbol{P} \\
\text { Value }\end{array}$ \\
\hline Contrast extravasation & 4.48 & 1.31 & 15.29 & $.017^{\mathrm{a}}$ \\
Midline shift $(\geq 5 \mathrm{~mm})$ & 13.77 & 1.54 & 123.49 & $.020^{\mathrm{a}}$ \\
GCS $\leq 8$ & 4.68 & 1.23 & 17.80 & $.024^{\mathrm{a}}$ \\
Age $>65 \mathrm{yr}$ & 4.61 & 1.00 & 21.33 & .050 \\
\hline
\end{tabular}

Note:-GCS indicates Glasgow Coma Scale score.

${ }^{a}$ Indicates statistically significant results $(P<.05)$.

extravasation, midline shift, and Glasgow Coma Scale score $\leq 8$ were independently associated with outcome, while there was a trend for patient age $(P=.0503)$ (Table 2). Contrast extravasation sensitivity, specificity, positive predictive value, negative predictive value, positive likelihood ratio, negative likelihood ratio, and area under the receiver operating characteristic analysis curve for the prediction of poor outcome were $77 \%, 71 \%, 67 \%, 80 \%, 2.62$, 0.33 , and 0.78 respectively.

\section{Per-Lesion Analysis}

A total of 112 hematomas were included in the per-lesion analysis (98 hematomas had imaging follow-up and 14 were drained). Eighty-three $(60 \%)$ traumatic intracerebral hematomas, 44 (32\%) subdural hematomas, and 11 (8\%) epidural hematomas were evaluated. Nine $(8 \%)$ hematomas showed significant progression. Twenty-two (33\%) traumatic intracerebral hematomas, $11(33 \%)$ subdural hematomas, and 8 (73\%) epidural hematomas showed at least 1 pattern of extravasation. Forty-one $(37 \%)$ hematomas showed contained extravasation, 30 (27\%) showed active extravasation, and 11 (8\%) hematomas showed isolated PCL. The latest contained extravasation, active extravasation, and PCL foci were identified at 16.0, 14.3 and 9.5 hours, respectively. Median (interquartile range) initial hematoma volume was $3.3 \mathrm{~mL}(0.80-11.5 \mathrm{~mL})$; absolute volume change, $+0.2 \mathrm{~mL}(-10.8-47.0 \mathrm{~mL})$; and percentage volume change, $+31 \%$ (-47\%-2298\%).

On-line Table 4 shows the predictive value of initial hematoma volume and extravasation patterns for the per-lesion end points. Contrast extravasation, including all extravasation patterns, was predictive of hematoma expansion, need for evacuation, and the combination of those 2 end points. An increasing number of extravasation foci had a graded prognostic value for the prediction of hematoma expansion and the combination of hematoma expansion and evacuation. When we examined specific extravasation subtypes, both extravasation patterns identifiable on CTA (ie, contained and active extravasation) were predictive of the end points and had graded prognostic value with increasing number of foci. Active extravasation showed a trend toward stronger association with these outcomes compared with contained extravasation. The presence of extravasation seen only on delayed imaging (postcontrast leakage) was not predictive of any of the end points. Finally, contrary to the per-patient analysis, in the perlesion multivariate analysis, extravasation remained significant (OR, 5.54; 95\% CI, 1.97-15.59; $P=.001$ ), while initial volume size showed only a trend toward prediction of the outcome (OR, 1.05; 95\% CI, 0.99-1.11; $P=.085)$.

\section{DISCUSSION}

Akin to prior nontraumatic ICH studies ${ }^{12-18,20-22,38}$ and a smaller cerebral contusion study, ${ }^{25}$ our results demonstrate the prognostic value of contrast extravasation in traumatic intracrananial injuries. Similarly, earlier MR imaging series evaluated "enhancement" of traumatic intra- and extra-axial hematomas, also suggesting a predictive value of extravasation in this setting but were limited by significantly smaller sample sizes. ${ }^{39,40}$ In comparison with prior traumatic extravasation studies, the present study included a larger population, evaluated both intra- and extraaxial hematomas, and examined different patterns of contrast extravasation.

Given the possibility of multiple hematomas in any given patient, we considered the sum of all intracranial hematomas in the per-patient analysis, while extravasation was considered positive if at least 1 hematoma showed this finding. Therefore, association between extravasation positivity and matched hematoma growth could not be directly made in the per-lesion analysis. However, the fact that extravasation is statistically associated with poor outcome indicates that it is a prognostic marker, regardless of the underlying mechanism. The per-lesion analysis confirms that hematomas showing extravasation are indeed associated with progression, which is likely the dominant mechanism by which extravasation influences the overall prognosis. However, it has also been shown in spontaneous ICHs that patients demonstrating contrast extravasation show increased permeability, even in the unaffected hemisphere, indicating a more diffuse permeability abnormality. ${ }^{26}$ Whether a similar phenomenon is also present in traumatic hematomas and potentially influences outcomes needs to be further studied.

Previous reports examining extravasation in spontaneous intracerebral hematomas have advocated the use of delayed imaging to maximize sensitivity for extravasation identification. ${ }^{12,15,22,23}$ Ederies et $\mathrm{al}^{15}$ showed that absolute and mean hematoma expansion were greater in CTA-negative PCL-positive patients compared with CTA- and PCL-negative patients. These results must, however, be measured against the decreased specificity of extravasation seen on delayed imaging and the overshadowing effect of extravasation identified on CTA (CTA-spot sign) over PCL for hematoma-growth prediction. ${ }^{15}$ Irrespective of potentially different pathomechanisms, ${ }^{15,25,30}$ the lower predictive value of PCL is likely related to its slower extravasation rate and is consequently associated with a lower risk of significant hematoma expansion, as shown in a recent CT perfusion study. ${ }^{26}$ Comparable with these findings, our results showed no prognostic value associated with PCL, though it is possible that we did not have enough statistical power to show a weaker association between PCL and the per-lesion outcomes. Despite this finding, delayed imaging might still have a role in the characterization of CTA-positive extravasation foci as shown by the stronger association of active extravasation with poor outcome compared with contained extravasation. The former is favored to represent uncontained active hemorrhage, whereas the latter is more likely to represent contained hemorrhage in the form of a pseudoaneurysm.

A potential major caveat of our finding is the difference in CTA/PCCT acquisition protocols across different centers, which 
can potentially mitigate the external validity of these results and mandate reproduction in other centers. For example, Huang et $\mathrm{al}^{25}$ did not identify any CTA-positive extravasation foci; all the observed extravasation foci were identified on the PCCT, and, contrary to our results regarding PCL, PCCT extravasation foci were significantly associated with hemorrhage progression (by using a different threshold of $>5 \mathrm{~mL}$ or $30 \%$ ), clinical deterioration, and the need for surgery.

We encountered a slightly higher rate of extravasation per patient (50\%) compared with $43 \%$ in the study by Huang et al. ${ }^{25}$ This difference is likely related to the inclusion of extra-axial hematomas in our study, especially epidural hematomas, which showed higher prevalence of extravasation.

Age and baseline Glasgow Coma Scale were the sole clinical factors associated with poor outcome in the multivariate analysis and are well-established predictors of poor outcome after traumatic brain injury. $2,4,9,31,33,41$ Serum glucose levels trended higher in the poor-outcome group, similar to a previous description. $^{32}$ Initial hematoma volume $e^{2,3,6,9,42,43}$ and midline shift $^{35,36,42-44}$ are potent predictors of intracranial hematoma progression and poor outcome but have also been shown to be collinear. ${ }^{42,45}$ Contrast extravasation could, therefore, potentially offer additional information not provided by NCCT, though this would need to be assessed by directly comparing NCCT with CTA/PCCT. Because admission NCCT was often performed at different time points than CTA/PCCT, we did not perform this comparison, given the potential for significant interval change in hematoma characteristics. Among signs of active hemorrhage, the "swirl sign" is possibly the closest NCCT equivalent to contrast extravasation. ${ }^{46}$ However, this sign is not standardized, and interobserver agreement is unknown. In a study of contrast extravasation within primary ICHs, Kim et $\mathrm{al}^{23}$ found the swirl sign to be predictive of mortality in univariate analysis but only extravasation remained significant in subsequent multivariate analysis. The predictive value of this sign in extra-axial hematomas has also been questioned in another study. ${ }^{10}$

A shorter time from trauma to CTA/PCCT was associated with the presence of contrast extravasation, similar to findings in prior reports. ${ }^{12,15,20,23}$ Similarly, shorter trauma-to-CTA/PCCT time was a predictor of the combined outcome in the univariate analysis, also consistent with previous studies of traumatic and nontraumatic intracranial hematomas. ${ }^{6,12,23}$ Despite this time-sensitive nature of contrast extravasation, its predictive value remained significant after controlling for trauma to imaging time in the multivariate analysis, supporting the added value of this sign regardless of the timing of the examination, as shown previously. ${ }^{12,19,23}$

In the per-patient analysis, extravasation showed a trend toward prediction of poor outcome when controlling for initial total hematoma volume. Conversely, in the per-lesion analysis, contrast extravasation was independent of initial hematoma volume, while the latter became nonsignificant in the multivariate analysis. In addition to the lower statistical power in the per-patient compared with the per-lesion analysis, the difference between these 2 analyses could potentially be explained by the variation in the end points: stronger prediction of total hematoma volume burden for overall poor prognosis (espe- cially mortality prediction) but stronger predictive value of contrast extravasation for individual hematoma growth or evacuation.

While the $\mathrm{ABC} / 2$ volume calculation method is widely used in the spontaneous ICH and trauma literature, we elected for computer-aided planimetry, given the greater error of the former technique $^{47}$ and the improved performance of planimetry for irregularly shaped hematomas. ${ }^{48}$ There is no consistent definition for hematoma expansion in the trauma and spontaneous ICH literature. Expansion thresholds previously evaluated include any growth, ${ }^{3} 20 \%,{ }^{22} 25 \%,{ }^{5,49} 30 \%,{ }^{2} 30 \%$ or $5 \mathrm{~mL},{ }^{25} 30 \%$ or 6 $\mathrm{mL},{ }^{12,14,15,17}$ and $33 \%{ }^{7,19}$ To ensure the clinical significance of our results, we decided to use a stricter threshold in accordance with other spontaneous and traumatic ICH studies. ${ }^{8,16,50,51}$ In the absence of a widely accepted cutoff value for subdural hematoma and epidural hematoma expansion, the same definition was used for extra-axial hematomas.

In our modified definition of the spot sign, we allowed the possibility of connection between the contrast extravasation focus and an outside vessel in extra-axial hematomas, given the known occurrence of bridging vein, cortical artery, or meningeal artery rupture in epidural and subdural hematomas. ${ }^{52-54}$

The limitations of our study include the relatively small sample size and retrospective nature, including potential selection bias. To facilitate extravasation identification and volume measurement, we opted to exclude traumatic intracerebral hematomas of $<10 \mathrm{~mm}$ (volume of $<0.5 \mathrm{~mL}$ for a sphere) to limit errors secondary to volume averaging. ${ }^{55}$ Similarly, we used a threshold of 2-mm thickness for extra-axial hematoma because it was thought that distinction of normal vessels surrounding these hematomas from foci of extravasation would be difficult and because accurate planimetry could not be performed. Furthermore, these small hematomas are generally of lesser clinical significance. It has previously been shown in large trauma studies that small traumatic intracerebral hematomas are less at risk of expansion and show a smaller magnitude of expansion than larger hematomas. ${ }^{2,3,6,7}$

We did not specifically examine the role of extravasation in individual hematoma subtypes due to sample size restrictions. The relationship of CE and vasogenic edema prediction was also not evaluated but is known to be an important cause of secondary injury $^{56}$ and seems to be associated with the presence of contrast extravasation. ${ }^{25}$ Long-term prognosis, including Glasgow Outcome Scale scores, was not evaluated, because the focus of this study was primarily the acute in-hospital course. Mortality in trauma patients is often multifactorial. Although the prevalence of extracranial injuries between patients with or without extravasation was not significantly different, it is difficult to control for all the variables that may contribute to mortality in the trauma setting. Finally, we did not specifically calculate the excess dose of radiation secondary to CTA and PCCT. Using a similar protocol for patients with acute stroke, Mnyusiwalla et $\mathrm{al}^{57}$ observed that the addition of CTA and PCCT resulted in a supplemental mean estimated effective dose of $8.0 \mathrm{mSv}$ ( $5.4 \mathrm{mSv}$ for the CTA and 2.6 $\mathrm{mSv}$ for the PCCT), resulting in a dose almost 3 times that in NCCT.

AJNR Am J Neuroradiol 34:773-79 Apr 2013 www.ajnr.org 777 


\section{CONCLUSIONS}

Contrast extravasation appears to predict poor patient outcome in the setting of traumatic head injuries. When we evaluated individual hematomas, extravasation foci identified on CTA (contained and active extravasation) predicted hematoma expansion and the need for surgical evacuation, while foci appearing only on the delayed phase (PCL) were not predictive of these outcomes. A delayed phase following CTA may remain useful for the identification of expanding foci of extravasation but not necessarily to detect late-appearing extravasation foci. These results mirror the larger experience gained with spontaneous ICHs but should be validated in larger studies of traumatic head injuries. The additional value of extravasation compared with established NCCT prognostic signs needs to be further evaluated. Finally, the riskbenefit ratio of the increased radiation dose associated with a multimodal CT protocol compared with NCCT alone also requires further assessment.

Disclosures: Sean Symons-UNRELATED: Consultancy: Bayer, Comments: related to gadolinium not CT contrast.

\section{REFERENCES}

1. Maas AI, Stocchetti N, Bullock R. Moderate and severe traumatic brain injury in adults. Lancet Neurol 2008;7:728-41

2. Alahmadi H, Vachhrajani S, Cusimano MD. The natural history of brain contusion: an analysis of radiological and clinical progression. J Neurosurg 2010;112:1139-45

3. Chang EF, Meeker M, Holland MC. Acute traumatic intraparenchymal hemorrhage: risk factors for progression in the early post-injury period. Neurosurgery 2007;61:222-30, discussion 230-31

4. Compagnone C, d'Avella D, Servadei F, et al. Patients with moderate head injury: a prospective multicenter study of 315 patients. $\mathrm{Neu}$ rosurgery 2009;64:690-96, discussion 696-97

5. Oertel M, Kelly DF, McArthur D, et al. Progressive hemorrhage after head trauma: predictors and consequences of the evolving injury. J Neurosurg 2002;96:109-16

6. Narayan RK, Maas AI, Servadei F, et al. Progression of traumatic intracerebral hemorrhage: a prospective observational study. J Neurotrauma 2008;25:629-39

7. White CL, Griffith S, Caron JL. Early progression of traumatic cerebral contusions: characterization and risk factors. J Neurotrauma 2009;67:508-14, discussion 514-15

8. Yadav YR, Basoor A, Jain G, et al. Expanding traumatic intracerebral contusion/hematoma. Neurol India 2006;54:377-81

9. Chieregato A, Fainardi E, Morselli-Labate AM, et al. Factors associated with neurological outcome and lesion progression in traumatic subarachnoid hemorrhage patients. Neurosurgery 2005;56: 671-80, discussion 671-80

10. Subramanian SK, Roszler MH, Gaudy B, et al. Significance of computed tomography mixed density in traumatic extra-axial hemorrhage. Neurol Res 2002;24:125-28

11. Eastman AL, Chason DP, Perez CL, et al. Computed tomographic angiography for the diagnosis of blunt cervical vascular injury: is it ready for primetime? J Neurotrauma 2006;60:925-29, discussion 929

12. Delgado Almandoz JE, Yoo AJ, Stone MJ, et al. Systematic characterization of the computed tomography angiography spot sign in primary intracerebral hemorrhage identifies patients at highest risk for hematoma expansion: the spot sign score. Stroke 2009;40:29943000

13. Delgado Almandoz JE, Yoo AJ, Stone MJ, et al. The spot sign score in primary intracerebral hemorrhage identifies patients at highest risk of in-hospital mortality and poor outcome among survivors. Stroke 2010;41:54-60

14. Wada R, Aviv RI, Fox AJ, et al. CT angiography “spot sign” predicts hematoma expansion in acute intracerebral hemorrhage. Stroke 2007;38:1257-62

15. Ederies A, Demchuk A, Chia T, et al. Postcontrast CT extravasation is associated with hematoma expansion in CTA spot negative patients. Stroke 2009;40:1672-76

16. Li N, Wang Y, Wang W, et al. Contrast extravasation on computed tomography angiography predicts clinical outcome in primary intracerebral hemorrhage: a prospective study of 139 cases. Stroke 2011;42:3441-46

17. Park SY, Kong MH, Kim JH, et al. Role of “spot sign” on CT angiography to predict hematoma expansion in spontaneous intracerebral hemorrhage. J Korean Neurosurg Soc 2010;48:399-405

18. Delgado Almandoz JE, Kelly HR, Schaefer PW, et al. CT angiography spot sign predicts in-hospital mortality in patients with secondary intracerebral hemorrhage. J Neurointerv Surg 2012;4:442-47

19. Goldstein JN, Fazen LE, Snider R, et al. Contrast extravasation on CT angiography predicts hematoma expansion in intracerebral hemorrhage. Neurology 2007;68:889-94

20. Becker KJ, Baxter AB, Bybee HM, et al. Extravasation of radiographic contrast is an independent predictor of death in primary intracerebral hemorrhage. Stroke 1999;30:2025-32

21. Wang YH, Fan JY, Luo GD, et al. Hematoma volume affects the accuracy of computed tomographic angiography 'spot sign' in predicting hematoma expansion after acute intracerebral hemorrhage. Eur Neurol 2011;65:150-55

22. Hallevi H, Abraham AT, Barreto AD, et al. The spot sign in intracerebral hemorrhage: the importance of looking for contrast extravasation. Cerebrovasc Dis 2010;29:217-20

23. Kim J, Smith A, Hemphill JC 3rd, et al. Contrast extravasation on CT predicts mortality in primary intracerebral hemorrhage. AJNR Am J Neuroradiol 2008;29:520-25

24. Mauser HW, van Nieuwenhuizen O, Veiga-Pires JA. Is contrast-enhanced CT indicated in acute head injury? Neuroradiology 1984;26: 31-32

25. Huang AP, Lee CW, Hsieh HJ, et al. Early parenchymal contrast extravasation predicts subsequent hemorrhage progression, clinical deterioration, and need for surgery in patients with traumatic cerebral contusion. J Trauma 2011;71:1593-99

26. d'Esterre CD, Chia TL, Jairath A, et al. Early rate of contrast extravasation in patients with intracerebral hemorrhage. AJNR Am J Neuroradiol 2011;32:1879-84

27. Graeb DA, Robertson WD, Lapointe JS, et al. Computed tomographic diagnosis of intraventricular hemorrhage: etiology and prognosis. Radiology 1982;143:91-96

28. Appendix II: evaluation of relevant computed tomographic scan findings. Neurosurgery 2006;58:S2-62

29. Gazzola S, Aviv RI, Gladstone DJ, et al. Vascular and nonvascular mimics of the CT angiography "spot sign" in patients with secondary intracerebral hemorrhage. Stroke 2008;39:1177-83

30. Thompson AL, Kosior JC, Gladstone DJ, et al. Defining the CT angiography 'spot sign' in primary intracerebral hemorrhage. Can J Neurol Sci 2009;36:456-61

31. The Brain Trauma Foundation. The American Association of Neurological Surgeons. The Joint Section on Neurotrauma and Critical Care. Age. J Neurotrauma 2000;17:573-81

32. Liu-DeRyke X, Collingridge DS, Orme J, et al. Clinical impact of early hyperglycemia during acute phase of traumatic brain injury. Neurocritic Care 2009;11:151-57

33. The Brain Trauma Foundation. The American Association of Neurological Surgeons. The Joint Section on Neurotrauma and Critical Care. Glasgow Coma Scale score. J Neurotrauma 2000;17:563-71

34. Marshall LF, Marshall SB, Klauber MR, et al. The diagnosis of head injury requires a classification based on computed axial tomography. J Neurotrauma 1992;9 Suppl 1:S287-92

35. Maas AI, Hukkelhoven CW, Marshall LF, et al. Prediction of outcome in traumatic brain injury with computed tomographic characteristics: a comparison between the computed tomographic 
classification and combinations of computed tomographic predictors. Neurosurgery 2005;57:1173-82, discussion 1173-82

36. Maas AI, Steyerberg EW, Butcher I, et al. Prognostic value of computerized tomography scan characteristics in traumatic brain injury: results from the IMPACT study. J Neurotrauma 2007;24: 303-14

37. Steyerberg EW. Clinical Prediction Models: A Practical Approach to Development, Validation, and Updating. New York: Springer Science and Business Media; 2009

38. Murai $Y$, Takagi R, Ikeda $Y$, et al. Three-dimensional computerized tomography angiography in patients with hyperacute intracerebral hemorrhage. J Neurosurg 1999;91:424-31

39. Tomida M, Muraki M, Uemura K, et al. Postcontrast magnetic resonance imaging to predict progression of traumatic epidural and subdural hematomas in the acute stage. Neurosurgery 1998;43:6670, discussion 70-71

40. Takanashi $Y$, Shinonaga M. Magnetic resonance imaging for surgical consideration of acute head injury. J Clin Neurosci 2001;8: $240-44$

41. Steyerberg EW, Mushkudiani N, Perel P, et al. Predicting outcome after traumatic brain injury: development and international validation of prognostic scores based on admission characteristics. PLoS Med 2008;5:e165, discussion e165

42. Nelson DW, Nystrom H, MacCallum RM, et al. Extended analysis of early computed tomography scans of traumatic brain injured patients and relations to outcome. J Neurotrauma 2010;27:51-64

43. Jacobs B, Beems T, van der Vliet TM, et al. Computed tomography and outcome in moderate and severe traumatic brain injury: hematoma volume and midline shift revisited. J Neurotrauma 2011;28: 203-15

44. The Brain Trauma Foundation. The American Association of Neurological Surgeons. The Joint Section on Neurotrauma and Critical Care. Computed tomography scan features. J Neurotrauma 2000;17: 597-627

45. Yuh EL, Cooper SR, Ferguson AR, et al. Quantitative CT improves outcome prediction in acute traumatic brain injury. J Neurotrauma 2012;29:735-46

46. Zimmerman RA, Bilaniuk LT. Computed tomographic staging of traumatic epidural bleeding. Radiology 1982;144:809-12

47. Divani AA, Majidi S, Luo X, et al. The ABCs of accurate volumetric measurement of cerebral hematoma. Stroke 2011;42:1569-74

48. Huttner HB, Steiner T, Hartmann M, et al. Comparison of ABC/2 estimation technique to computer-assisted planimetric analysis in warfarin-related intracerebral parenchymal hemorrhage. Stroke 2006;37:404-08

49. Tong WS, Zheng P, Xu JF, et al. Early CT signs of progressive hemorrhagic injury following acute traumatic brain injury. Neuroradiology 2011;53:305-09

50. Broderick JP, Diringer MN, Hill MD, et al. Determinants of intracerebral hemorrhage growth: an exploratory analysis. Stroke 2007;38: 1072-75

51. Kazui S, Naritomi H, Yamamoto H, et al. Enlargement of spontaneous intracerebral hemorrhage. Incidence and time course. Stroke 1996;27:1783-87

52. Crooks DA. Pathogenesis and biomechanics of traumatic intracranial haemorrhages. Virchows Arch A Pathol Anat Histopathol 1991; 418:479-83

53. Cervós-Navarro J, Lafuente JV. Traumatic brain injuries: structural changes. J Neurol Sci 1991;103(suppl):S3-14

54. Maxeiner $\mathrm{H}$. Detection of ruptured cerebral bridging veins at autopsy. Forensic Sci Int 1997;89:103-10

55. Tsuchida $Y$, Therasse P. Response evaluation criteria in solid tumors (RECIST): new guidelines. Med Pediatr Oncol 2001;37:1-3

56. Bullock MR, Chesnut R, Ghajar J, et al. Surgical management of traumatic parenchymal lesions. Neurosurgery 2006;58:S25-46, discussion Si-iv

57. Mnyusiwalla A, Aviv RI, Symons SP. Radiation dose from multidetector row CT imaging for acute stroke. Neuroradiology 2009;51: $635-40$ 